Communications in Physics, Vol. 14, No. 2 (2004), pp. 84-89

\title{
MESONIC STIFF FLUID DISTRIBUTION IN BIANCHI TYPE SPACE-TIMES
}

\author{
G. MOHANTY, S. K. SAHU \\ P. G. Department of Mathematics, \\ Sambalpur University, Jyotivihar,Sambalpur-768018, India \\ P. K. SAHOO \\ Dept. of Engg. Mathematics, \\ Padmashree Krutartha Acharya College of Engineering, Bargarh - 768028
}

\begin{abstract}
The distributions of stiff perfect fluid coupled with zero mass scalar field in LRS Bianchi type-I \& Bianchi type-V space times are investigated. Some physical and geometrical properties of the models are discussed.
\end{abstract}

\section{INTRODUCTION}

The study of the nature of scalar fields without mass parameter interacting with stiff perfect fluid in Bianchi type space- times is a subject of interest due to its significant role in the description of the universe at the early stages of evolution. Patel [1] obtained the static and non-static plane symmetric solutions of the field equations in presence of zeromass scalar field. Singh and Deo [2] considered Robertson Walker metric and investigated the problem of zero-mass scalar field interactions in the presence of gravitational field with and without the source term in the wave equation. They derived the solution in presence of deceleration parameter and discussed the occurrence of "Big Bang" at the initial stage. Singh [3] considered the combined energy-momentum tensor for a perfect fluid, radially expanding the radiation with zero-mass scalar field and obtained five new analytic solutions in a spherically symmetric Einstein Universe. Reddy and Venkateswarlu [4] obtained spatially homogeneous and anisotropic Bianchi type $\mathrm{VI}_{0}$ cosmological model in Barber's second self creation theory of gravitation in vacuum and with stiff perfect fluid distribution. Shanti and Rao [5] obtained spatially homogenous and anisotropic Bianchi type II and III cosmological models in Barber's second self-creation theory of gravitation both in vacuum and in the presence of stiff fluid distribution. Mohanty and Sahu [6] studied the problem of inhomogeneous anisotrpic locally rotationally symmetric [henceforth referred as LRS] Bianchi type I space-time with perfect fluid and obtained exact solutions of the field equations when the metric potentials are functions of cosmic time ' $t$ ' only. Pradhan et al. [7] derived the field equations in LRS Bianchi type I spacetime in the presence of mesonic perfect fluid and solved the field equations considering a particular case. Recently Mohanty et al. [8] obtained a class of exact solutions of Einstein's field equations with attractive massive scalar field in LRS Bianchi type I space time and showed that how the dynamical importance of scalar field and the shear change in the course of evolution.

In section 2 we derived Einstein's field equations for stiff perfect fluid coupled with zero mass scalar field in the space-time described by LRS Bianchi type I metric. In section 3 we set up Einstein's field equations for mesonic stiff perfect fluid distribution in Bianchi 
type V space-time. We found that the source density of scalar meson field vanishes for all the models discussed here. We mentioned some physical properties of the solutions in section 4 .

\section{LRS BIANCHI TYPE I SPACE-TIME}

We considered here the LRS Bianchi type-I metric in the form

$$
d s^{2}=-d t^{2}+A^{2} d x^{2}+B^{2}\left(d y^{2}+d z^{2}\right)
$$

where $A$ and $B$ are functions of cosmic time $t$ only. The Einestein's field equations in presence of perfect fluid and zero mass scalar field are given by

$$
G_{i j} \equiv R_{i j}-\frac{1}{2} g_{i j} R=-8 \pi\left(T_{i j}^{p}+T_{i j}^{v}\right)
$$

where

$$
T_{i j}^{p}=(\rho+p) U_{i} U_{j}+p g_{i j}
$$

is the energy momentum tensor for a perfect fluid together with

$$
g_{i j} U^{i} U^{j}=-1
$$

where $U^{i}$ is the four velocity vector of the fluid, $p$ and $\rho$ are the proper pressure and energy density of the fluid distribution respectively and

$$
T_{i j}^{v}=\frac{1}{4 \pi}\left[V_{, i} V_{, j}-\frac{1}{2} g_{i j}\left(V_{, a} V^{, a}\right)\right]
$$

Where $T_{i j}^{v}$ is the stress energy tensor corresponding to zero mass scalar field.

The Klein Gordon equations corresponding to the scalar field $V$ are given by

$$
g^{i j} V_{; i j}=\sigma(t)
$$

where $\sigma$ is the source density of the scalar meson field. Here the comma and semicolon denote ordinary and covariant differentiations respectively and the units are chosen such that $G=1=c$. The explicit forms of the field equations (2) for the metric (1) may be written as

$$
\begin{gathered}
\frac{2 B_{44}}{B}+\frac{B_{4}^{2}}{B^{2}}=-\left(8 \pi p+V_{4}^{2}\right) \\
\frac{B_{44}}{B}+\frac{A_{4} B_{4}}{A B}+\frac{A_{44}}{A}=-\left(8 \pi p+V_{4}^{2}\right)
\end{gathered}
$$

and

$$
\frac{2 A_{4} B_{4}}{A B}+\frac{B_{4}^{2}}{B^{2}}=8 \pi \rho+V_{4}^{2}
$$

Hereafterwards the suffix 4 after a field variable represents ordinary differentiation with respect to $t$. The conservation equations

$$
T_{; j}^{i j}=0
$$


for $i=4$ yields

$$
\frac{A_{4}}{A}+\frac{2 B_{4}}{B}=\frac{-\rho_{4}}{p+\rho}
$$

For the metric (1), equation (6) reduces to

$$
V_{44}+\left(\ln \left(A B^{2}\right)\right)_{4} V_{4}=\sigma
$$

Here we intend to derive the exact solutions of the field equations (7)-(15) with the help of the scale transformations i.e.

$$
A=e^{\alpha}, B=e^{\beta}, d t=A B^{2} d T
$$

The field equations (7) - (9) and equations (11) and (15) reduce to

$$
\begin{gathered}
2 \beta^{\prime \prime}-2 \alpha^{\prime} \beta^{\prime}-\beta^{\prime^{2}}=-\left(8 \pi p \cdot e^{2 \alpha+4 \beta}+V^{\prime 2}\right) \\
\beta^{\prime \prime}-{\beta^{\prime}}^{2}+\alpha^{\prime} \beta^{\prime \prime}-\beta^{\prime 2}+\alpha^{\prime \prime}-2 \alpha^{\prime} \beta^{\prime}=- \\
2 \alpha^{\prime} \beta^{\prime}+\beta^{\prime 2}=8 \pi \rho \cdot e^{2 \alpha+4 \beta}+V^{\prime 2} \\
\alpha^{\prime}+2 \beta^{\prime}=\frac{-\rho^{\prime}}{p+\rho}
\end{gathered}
$$

and

$$
\frac{V^{\prime \prime}}{e^{2(\alpha+2 \beta)}}=\sigma
$$

Hereafterwards the prime stands for $\frac{d}{d T}$.

From equations (14) and (15) we get

$$
\alpha^{\prime \prime}=\beta^{\prime \prime}
$$

which yields

$$
\alpha=\beta+K_{1} T+K_{2}
$$

where $K_{1}$ and $K_{2}$ are arbitrary constants.

From equations (14) and (16) we get

$$
\beta^{\prime \prime}=-4 \pi(p-\rho) e^{2 \alpha+4 \beta}
$$

In order to avoid the under determinacy because of six unknowns with five field equations, we consider here the case of stiff perfect fluid with $p=\rho$. Now equation (21) yields

$$
\beta=K_{4} T+K_{5}
$$

where $K_{4}$ and $K_{5}$ are arbitrary constants.

Substituting $p=\rho$ in equation (17) we get

$$
\rho=\frac{K_{6}^{2}}{e^{2 \alpha+4 \beta}}
$$


where $K_{6}$ is an integration constant.

Using equations (20), (22) and (23) in equation (16) we get

$$
V=K_{7} T+K_{8}
$$

where $K_{7}=\left(3 K_{4}^{2}-8 \pi K_{6}^{2}\right)^{\frac{1}{2}}=$ constant and $K_{8}$ is an integration constant.

Using equation (24) in equation (18) we obtain

$$
\sigma=0
$$

With the help of equations (20) and (22) the pressure and energy density of the model are obtained as

$$
\rho=p=\frac{K_{6}^{2}}{e^{2 T\left(3 K_{4}+K_{1}\right)+6 K_{5}+2 K_{2}}}
$$

The corresponding stiff fluid model can be written in the form

$$
d s^{2}=e^{2\left(K_{3} K_{4}+K_{1}\right) T} d T^{2}-e^{2\left(K_{4}+K_{1}\right) T} d X^{2}-e^{2 K_{4} T}\left(d Y^{2}+d Z^{2}\right)
$$

\section{BIANCHI TYPE - V SPACE TIME}

Here we consider the Bianchi type- $\mathrm{V}$ space-time in the form

$$
d s^{2}=-d t^{2}+e^{2 \alpha} d x^{2}+e^{2(x+\beta)} d y^{2}+e^{2(x+\gamma)} d z^{2}
$$

where $\alpha, \beta$ and $\gamma$ are functions of cosmic time " $t$ " only.

By the use of commoving coordinate system the field equations (2) for the metric (28) can be written as

$$
\begin{aligned}
\beta_{44}+\beta_{4}^{2}+\gamma_{44}+\gamma_{4}^{2}+\beta_{4} \gamma_{4}-e^{-2 \alpha} & =-\left(8 \pi p+V_{4}^{2}\right) \\
\gamma_{44}+\gamma_{4}^{2}+\alpha_{44}+\alpha_{4}^{2}+\gamma_{4} \alpha_{4}-e^{-2 \alpha} & =-\left(8 \pi p+V_{4}^{2}\right) \\
\alpha_{44}+\alpha_{4}^{2}+\beta_{44}+\beta_{4}^{2}+\alpha_{4} \beta_{4}-e^{-2 \alpha} & =-\left(8 \pi p+V_{4}^{2}\right) \\
\alpha_{4} \beta_{4}+\beta_{4} \gamma_{4}+\gamma_{4} \alpha_{4}-3 e^{-2 \alpha} & =8 \pi \rho+V_{4}^{2}
\end{aligned}
$$

and

$$
2 \alpha_{4}-\beta_{4}-\gamma_{4}=0
$$

The conservation equation (10) for the metric (28) may be written as

$$
\alpha_{4}+\beta_{4}+\gamma_{4}=-\frac{\rho_{4}}{p+\rho}
$$

The Klein Gordon equation (6) for the metric (28) may be written as

$$
V_{44}+\left(\alpha_{4}+\beta_{4}+\gamma_{4}\right) V_{4}=\sigma
$$

Hereafterwards the suffix 4 after a field variable represents exact differentiation with respect to time. 
Equations (29) - (31) yield

$$
\frac{\alpha_{44}-\beta_{44}}{\alpha_{4}-\beta_{4}}=\frac{\beta_{44}-\gamma_{44}}{\beta_{4}-\gamma_{4}}=\frac{\gamma_{44}-\alpha_{44}}{\gamma_{4}-\alpha_{4}}=-\left(\alpha_{4}+\beta_{4}+\gamma_{4}\right)
$$

Comparing equation (36) with equation (33), we get

$$
\alpha_{4}=\beta_{4}=\gamma_{4}
$$

Using equation (37) in equations (29)-(32), equations (34)-(35), we get

$$
\begin{gathered}
2 \alpha_{44}+3 \alpha_{4}^{2}-e^{-2 \alpha}=-\left(8 \pi p+V_{4}^{2}\right) \\
3 \alpha_{4}^{2}-3 e^{-2 \alpha}=8 \pi \rho+V_{4}^{2} \\
3 \alpha_{4}=-\frac{\rho_{4}}{p+\rho} \\
V_{44}+3 \alpha_{4} V_{4}=\sigma
\end{gathered}
$$

Since the field equations are highly non linear in nature, we consider the case

$$
p=\rho \text { and } \sigma=0
$$

Now equation (38) and equation (39) yield

$$
e^{2 \alpha}=\left(t+a_{1}\right)^{2}
$$

where $a_{1} \quad(>0)$ is an arbitrary constant.

Substitution of equation (42b) in equation (43) in equation (41) yields

$$
V=-\frac{a_{2}}{2\left(t+a_{1}\right)^{2}}+a_{3}
$$

where $a_{2}(\neq 0)$ and $a_{3}$ are arbitrary constants.

Substituting equations (43)-(44) in equations (38)-(39), we get

$$
\rho(=p)=-\frac{a_{2}^{2}}{8 \pi\left(t+a_{1}\right)^{6}}
$$

Thus in this case the stiff fluid model can be written in the form

$$
d s^{2}=-d t^{2}+\left(t+a_{1}\right)^{2}\left[d x^{2}+e^{2 x}\left(d y^{2}+d z^{2}\right)\right]
$$

\section{PHYSICAL INTERPRETATION OF THE SOLUTIONS}

From equations (26) we observe that the energy density and pressure decrease with time and tends to zero as $T \rightarrow \infty$. Equations (24) show that the scalar field V increases with time and tends to $\infty$ as $T \rightarrow \infty$. At time $T=0$, the scalar field is found to be a constant. 
The magnitude of scalar expansion $\theta$, shear $\sigma^{2}$ and the spatial volume (Vol.) for the model (27) are given by

$$
\begin{gathered}
\theta=3 K_{4}+K_{1} \\
\sigma^{2}=\frac{K_{1}^{2}}{e^{\left(6 K_{4}+2 K_{1}\right) T+6 K_{5}+2 K_{2}}}
\end{gathered}
$$

and

$$
\text { Vol. }=\left\{\left(K_{4}+K_{1}\right) T+K_{5}+K_{2}\right\}\left(K_{4} T+K_{5}\right)^{2}
$$

Here $\frac{\sigma^{2}}{\theta^{2}} \rightarrow 0$ as $T \rightarrow \infty$ which implies that the model approaches isotropy for large value of $T$. Equation (49) shows the anisotropic expansion of the universe with time.

From equations (45) we observe that the energy density and pressure decrease with time and tends to zero as $t \rightarrow \infty$. Equations (44) show that the scalar field V changes with time and at time $\mathrm{t}=0$ the scalar field is found to be a constant.

The scalar expansion $\theta$, shear $\sigma^{2}$ and the spatial volume (Vol.) for the model (46) are obtained as

and

$$
\begin{gathered}
\theta=\frac{3}{t+a_{1}} \\
\sigma^{2}=\frac{6}{\left(t+a_{1}\right)^{2}} \\
\text { Vol. }=\log \left(t+a_{1}\right)^{3}
\end{gathered}
$$

Here $\frac{\sigma^{2}}{\theta^{2}}=\frac{2}{3}$ being independent of cosmic time implies that the model does not approach isotropy. As in the preceding case this model is also expanding in nature, but the expansion is decelerating. Equation (54c) shows the isotropic expansion of the Universe with time.

\section{ACKNOWLEDGEMENT}

The authors are very much thankful to the referee for his valuable suggestions for the improvement of the paper.

\section{REFERENCES}

1. Patel L. K., Tensor N.S., 29 (1975) 237.

2. Singh R. T. and Deo S. Acta Physica Hungarica, 59(3-4) (1986) 321.

3. Singh K. M., Int. J. Theor. Phys., 27 (3) (1988) 345.

4. Reddy D.R.K. and Venkateswarlu R., Astrophys. and Space Sci., 155 (1989) 135.

5. Shanthi K. and Rao V. U. M., Astrophys. and Space Sci., 179 (1991) 147.

6. Mohanty G. and Sahu S. K., Theo. Appl. Mech., 26 (2001) 59.

7. Pradhan A. Tiwari K. L. and Beesham A., Indian J. Pure Appl. Math., 32 (6) (2001) 789.

8. Mohanty G. Sahu S. K. and Sahoo P. K., Theo. App. Mech., (2002) Communicated. 
